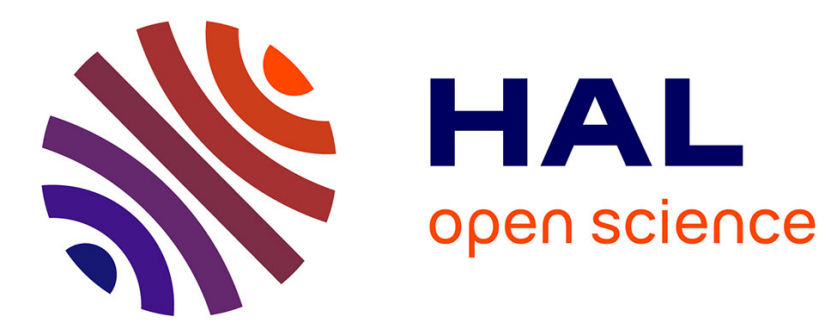

\title{
FLASHthe first soft x-ray free electron laser (FEL) user facility
}

J Feldhaus

\section{To cite this version:}

J Feldhaus. FLASHthe first soft x-ray free electron laser (FEL) user facility. Journal of Physics B: Atomic, Molecular and Optical Physics, 2010, 43 (19), pp.194002. 10.1088/0953-4075/43/19/194002 . hal-00569835

\section{HAL Id: hal-00569835 https://hal.science/hal-00569835}

Submitted on 25 Feb 2011

HAL is a multi-disciplinary open access archive for the deposit and dissemination of scientific research documents, whether they are published or not. The documents may come from teaching and research institutions in France or abroad, or from public or private research centers.
L'archive ouverte pluridisciplinaire HAL, est destinée au dépôt et à la diffusion de documents scientifiques de niveau recherche, publiés ou non, émanant des établissements d'enseignement et de recherche français ou étrangers, des laboratoires publics ou privés. 


\title{
FLASH - The first soft X-ray FEL user facility
}

\author{
J Feldhaus \\ Deutsches Elektronen-Synchrotron DESY, 22603 Hamburg, Germany \\ Email: josef.feldhaus@desy.de
}

\begin{abstract}
The free electron laser FLASH at DESY in Hamburg is the first X-ray FEL ever built. Many new developments were necessary in order to exploit the unique properties of this novel light source for scientific experiments. The facility has constantly been improved and several major upgrades have been made or are currently underway. The article reviews the main characteristics of the user facility as well as the major developments and upgrades.
\end{abstract}

PACS numbers: $41.60 \mathrm{Cr}, 29.20 . \mathrm{Ej}$

\section{Introduction}

FLASH, the free electron laser (FEL) facility at DESY in Hamburg, is the first FEL ever built for the vacuum-ultraviolet (VUV) and soft X-ray region. Its conceptual design and a science case were developed in 1994-5 with the enthusiastic support of a broad user community that had experience with synchrotron radiation and optical lasers [1]. In the following years, during the proof-of-principle experiments on the TESLA Test Facility (TTF) FEL [2], it became apparent that the unprecedented peak brightness of this new laser light source would have significant impact not only on the physics that would occur in the beam focus, but also on the way one would do the experiments and on the design of beam lines, optics, photon diagnostics, detectors and data acquisition. Many experiments, including photon diagnostics, would be single-shot measurements, and the fluctuating pulse properties would make it necessary to monitor and associate them, pulse by pulse, with the experimental data.

Consequently, programmes were started at DESY to develop pulse resolved photon diagnostics, to measure damage thresholds of relevant optical materials, and to build an optical femtosecond laser system with the same burst-like pulse structure as the FEL for two-colour pump-probe experiments. Likewise, user groups started to build special instrumentation for experiments at FLASH, after their proposals had been evaluated by an independent, international peer review panel in 2002. The technical requirements of these first sixteen projects were taken into account in the design and construction of the beamlines and the layout of the experimental area.

FLASH started regular user operation in August 2005, immediately after the preliminary commissioning of the FEL at a wavelength of $\sim 32 \mathrm{~nm}$ and of the first photon beamlines [3, 4]. At this time the accelerator was still incomplete: only five out of six accelerator modules were installed, limiting the maximum electron beam energy to about $700 \mathrm{MeV}$, rather than the design energy of 1 $\mathrm{GeV}$; and the $3.9 \mathrm{GHz}$ section in the injector was still missing. Therefore, wavelengths below $10 \mathrm{~nm}$ were not accessible, and the FEL pulses were shorter than originally anticipated because only a small fraction of the electron bunch contributed to the FEL process. The early start of regular user operation, typically in four-week blocks interleaved with a few weeks for machine and FEL studies for improving the performance of the light source, turned out to be very efficient. Not only the light source, but also the beam lines and photon diagnostics as well as all the new user experiments had to go through a commissioning and learning phase. There was a very close and fruitful interaction between all the teams involved, and the intensive support of the new user groups by the facility, based on the experience gained at TTF, made the start-up phase of the FLASH user facility rather efficient and successful. Reference [5] highlights a selection of first scientific results obtained at FLASH. 
This article describes the photon beam transport and the experimental area, the developments for time resolved experiments and photon diagnostics, as well as major facility upgrades.

\section{Photon beam transport and experimental area}

The design of the photon beam transport and the experimental area was mainly based on two considerations: the requirements of different classes of scientific experiments and possible damage of optical components due to the high peak power of the FEL beam. In the beginning, it was not clear if mirrors would survive the FEL beam and, therefore, the experiments would need to be done in the accelerator tunnel, just like the first experiments on the TTF FEL. This would have been very cumbersome and inefficient, but fortunately, thanks to the World Exposition EXPO 2000, the experimental building could be constructed outside the PETRA tunnel and the previous DESY premises, increasing the distance of the first mirror to the FEL undulator from $\sim 5 \mathrm{~m}$ to $\sim 40 \mathrm{~m}$. The construction of the accelerator tunnel and the new experimental hall were ready for the exposition in the beginning of the year 2000, i.e. before first lasing at 100 nm wavelength.

\subsection{Design criteria for FEL optics}

Before the TTF FEL became operational there were hardly any data available on damage of optics in the extended ultraviolet (EUV). In order to get a rough estimate for the interaction of the GW FEL pulses with matter, an average energy absorbed per atom during an FEL pulse was calculated by multiplying the photon density (i.e. the number of photons per pulse divided by the beam cross section) with the atomic photoionization cross section [6]. Figure 1 shows the resulting curves for B, $\mathrm{C}, \mathrm{Si}, \mathrm{Ni}$ and $\mathrm{Au}$ in the photon energy range below $1000 \mathrm{eV}$, assuming a constant photon density of 2 $\cdot 10^{17}$ photons $/ \mathrm{cm}^{2}$. Such densities are typical at FLASH for long wavelengths in the direct beam behind the undulator or, for shorter wavelengths, in a moderate beam focus of a few tens of $\mu \mathrm{m}$ diameter. Obviously, it is easily possible in this spectral range to ionise each atom in the beam and to convert solid material into hot dense plasma, also referred to as "warm, dense matter" [7].

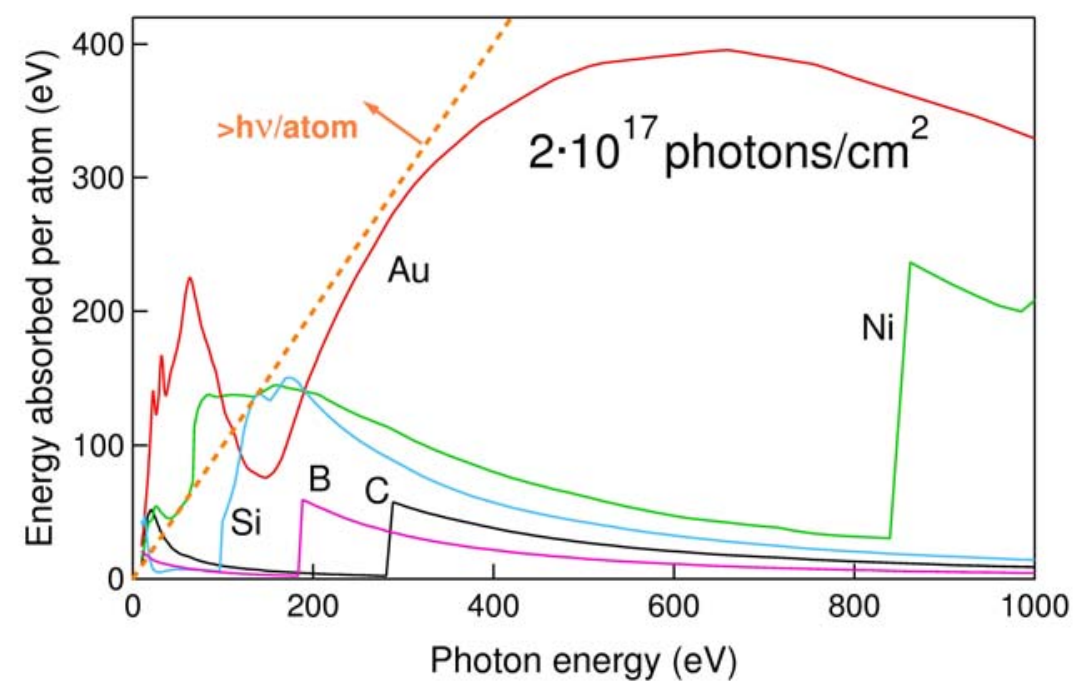

Figure 1. Average energy absorbed by boron, carbon, silicon, nickel and gold atoms in a FEL beam, calculated by multiplying the atomic photoionization cross sections [6] with a constant photon density of $2 \cdot 10^{17}$ photons $/ \mathrm{cm}^{2}$. This photon density corresponds to a $100 \mu \mathrm{J}$ pulse at $100 \mathrm{eV}$ photon energy focused to a spot of $\sim 50 \mu \mathrm{m}$ diameter. On the dashed line the product of cross section and photon density is 1 . 
Carbon is the material of choice for optical coatings at FLASH because it has a low photoionization cross section and no absorption edges in the entire spectral range of the fundamental frequency. Sputter-deposited amorphous carbon coatings of high optical quality were developed and characterized in collaboration with the research centre GKSS [8]. The measured reflectivity was close to the theoretical value ( $95 \%$ for $2^{\circ}$ grazing angle of incidence), and experiments at the TTF FEL near $100 \mathrm{~nm}$ wavelength gave an estimate of 70 $\mathrm{mJ} / \mathrm{cm}^{2}$ for the damage threshold. Based on these results, the angles of incidence for the plane deflecting mirrors were chosen at $2^{\circ}$ such that the maximum fluence would be smaller than 20 $\mathrm{mJ} / \mathrm{cm}^{2}$. The damage studies initiated at TTF have led to an international collaboration that has systematically investigated the damage of materials under intense FEL radiation $[9,10]$. This work is still ongoing: The experiments have now been extended to Angstrom wavelengths at LCLS, and long-term degradation is studied at FLASH.

\subsection{Layout of the experimental area}

Based on the experimental requirements five ultra-high vacuum (UHV) beam lines (BL1-3, PG1 and PG2) were constructed at FLASH. They are indicated as red lines in the photograph of the experimental area (Figure 2). A detailed description of the user facility can be found in reference [11].

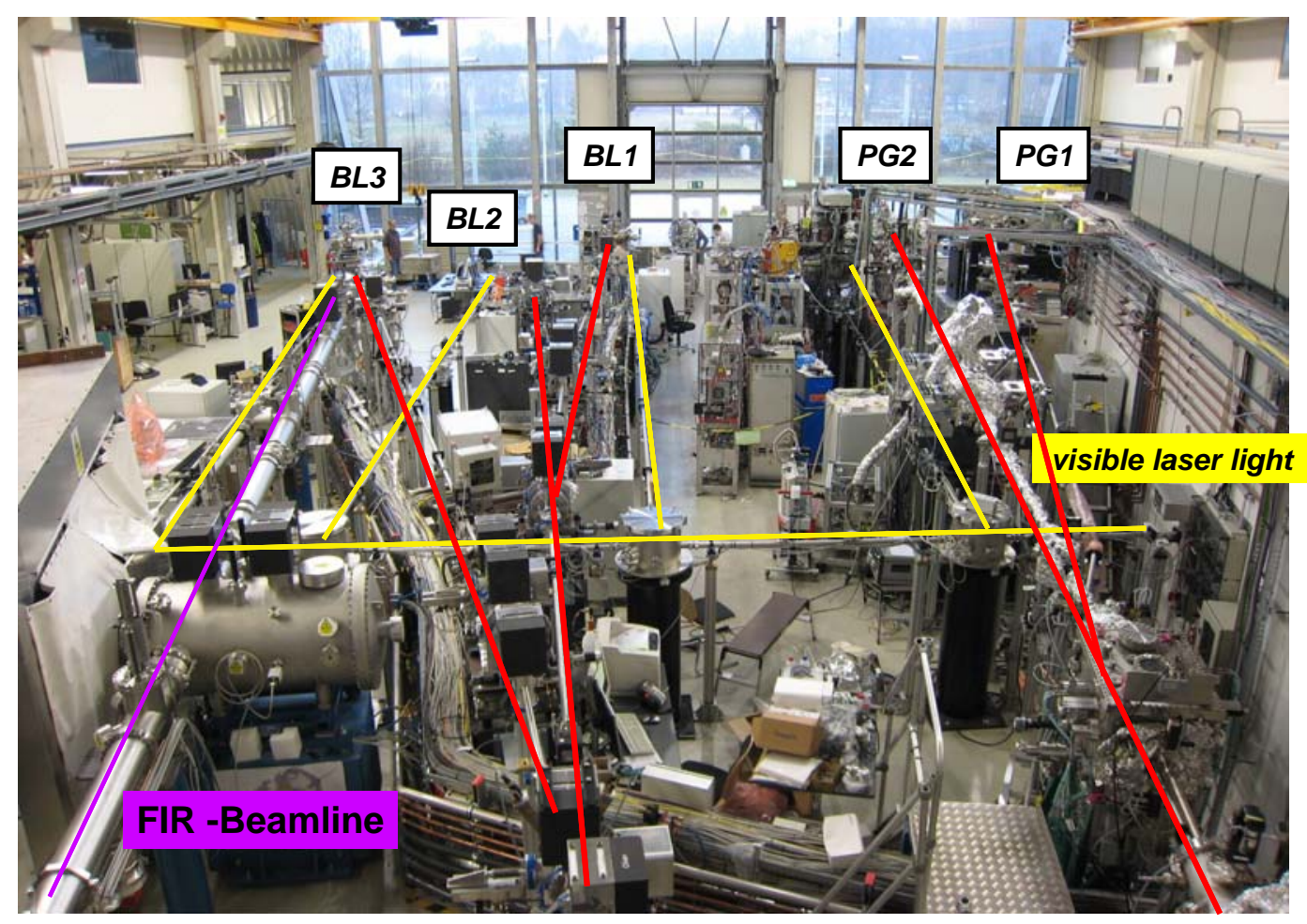

Figure 2. Photograph of the experimental area. The FEL beam can be switched to five experimental stations (in the background close to the window) by moving plane mirrors. Similarly, a synchronised optical laser beam can be transported in vacuum to each of the stations by switching plane mirrors which are mounted in the four cylindrical vacuum vessels in the foreground. The optical laser is located in a hutch on the right just outside the picture.

The FEL beam can be switched from one station to another simply by moving one or two plane mirrors in or out of the beam. Beam line BL1 provides a $\sim 100 \mu \mathrm{m}$ focus using a toroidal mirror with $10 \mathrm{~m}$ focal distance. Beam lines BL2 and BL3 both provide $\sim 20 \mu \mathrm{m}$ beam spots produced by ellipsoidal mirrors with $2 \mathrm{~m}$ focal distance. The ellipsoidal mirrors can be retracted such that the 
unfocused FEL beam can be used for experiments or special focusing optics integrated in the experimental system to achieve $\mu \mathrm{m}$ or even sub- $\mu \mathrm{m}$ beam spots on the sample (see for example [12, 13]). The PG1 and PG2 stations are served by a high-resolution plane-grating monochromator [14, 15]. This device was designed for high resolution spectroscopy requiring relative spectral bandwidths of the order $10^{-4}$ or below, while the natural bandwidth of the FEL pulses is $\sim 0.5-1 \%$. However, the monochromator may also be used to suppress the dominant fundamental frequency for experiments exploiting higher harmonic radiation. PG1 was specifically designed for inelastic scattering experiments and includes a micro-focusing optical system for the radiation coming from the monochromator, a special experimental chamber and a high-resolution, two-stage grating spectrometer with a 2D pixel detector.

All experimental stations can be combined with an optical laser that is synchronised with the FEL [16, 17]. Evacuated beamlines guide the laser beam to the experimental stations (indicated by yellow lines in figure 2). The laser beam can be switched by moving plane mirrors in or out, just like the FEL beam. Suitable delay lines in the laser hutch are used to adjust the timing between the FEL and the optical laser pulses. In addition, short and coherent far-infrared (FIR) pulses are available at the BL3 station [18].

All beam lines except PG1 have a standard UHV interface to which the user experiments can be connected. In order to use the FEL beam efficiently, at least two experiments are set up at a time at different stations between which the FEL beam is usually switched once a day. In general, the experimental equipment is brought by the user groups. So far, 30 different experimental systems have been used. Due to the little beam time available for each project, also the users groups are trying to work as efficient as possible by collaborating with other teams and by building special instruments that can be used for several projects, avoiding constant set-up and removal of equipment.

\section{Developments for time-resolved experiments}

Large efforts have been made to exploit the femtosecond FEL pulses for the study of ultrafast phenomena. This work started already in 2000 with a small network funded by the European Commission under their $5^{\text {th }}$ Framework Programme. The burst-mode laser system and the pump-probe facility resulting from this collaboration has been described in references [16] and [17]. This laser system is routinely available for user experiments at all experimental stations (see above). However, the laser pulse duration is $\sim 120$ fs, i.e. about an order of magnitude longer than that of the FEL pulses, because it was adapted to the original design parameters of FLASH where FEL pulses of $\sim 200 \mathrm{fs}$ duration were anticipated. In order to fully exploit the short FEL pulses, a new high-power laser system is under development which will deliver sub-10 fs pulses at high repetition rate using fibrelaser pumped optical parametric chirped pulse amplification [19].

The main technical challenge for achieving $10 \mathrm{fs}$ time resolution in pump-probe experiments is the synchronisation between the optical laser and the FEL. The major problem is the short-term jitter in the arrival time of the FEL pulses, another are long-term drifts due to the distribution of the master clock signal to all relevant components of the facility over distances of up to $\sim 300 \mathrm{~m}$. In agreement with early expectations based on the measured energy variation of the electron bunches at TTF, the short-term timing jitter was measured at $\sim 500$ fs FWHM by using a cross-correlation experiment (Figure 3). It arises from different path lengths - and hence travelling times - of the electron bunches through the magnetic chicanes of the bunch compression system due to small energy variations from bunch to bunch. 


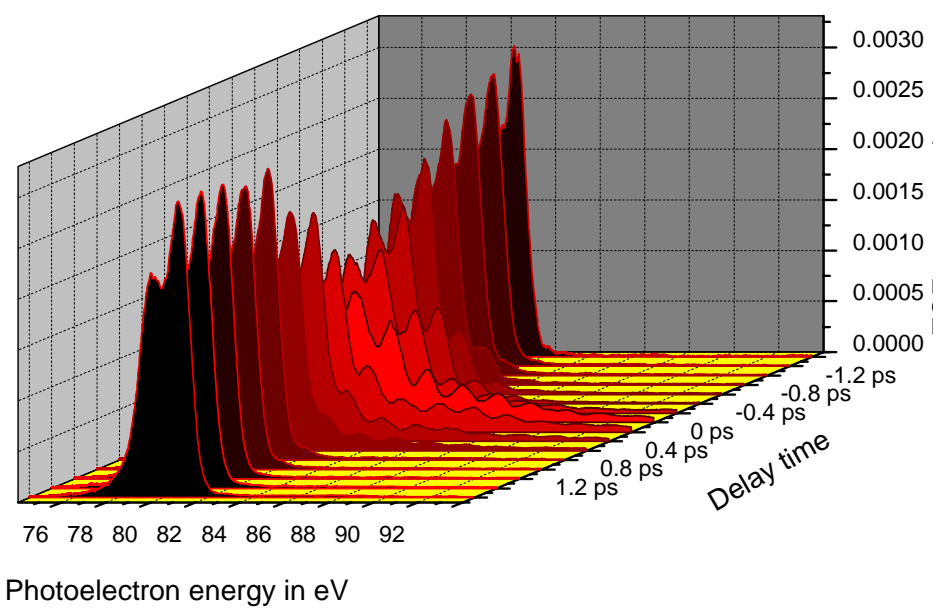

Figure 3. Series of Xenon $5 p$ photoelectron spectra measured at $13 \mathrm{~nm}$ wavelength at FLASH for different delay times between the FEL pulse and the optical laser pulse. Strong side bands appear when the photoelectrons are created in the presence of the optical laser pulse [17].

Three different routes have been followed at FLASH to improve this situation: (i) reduction of timing jitter and drifts; (ii) measurement of the relative timing between FEL and optical laser pulses by suitable diagnostics; (iii) combination of photon pulses that are strictly synchronised with each other. Regarding the first point, a number of technical developments and proof-of-principle experiments have been made during the last years that have resulted in a completely new design of the synchronisation system of the FLASH facility [20]. It will be based on low-jitter fibre laser master oscillators, stabilized fibre links and fast bunch arrival time monitors. The major part of this system has now been installed at FLASH and will be commissioned in the coming months.

In parallel, diagnostic systems have been developed and integrated in the facility that measure the relative timing of the FEL with respect to the optical laser pulse by pulse [17]. The short-term timing jitter is routinely measured by an electro-optical sampling system where part of the laser light is transported via an optical fibre to the accelerator and sent through an electro-optical crystal near the electron beam. The resolution of this system is $\sim 100 \mathrm{fs}$. Long-term drifts of the optical laser are corrected by using a streak camera in the laser hutch on the entrance slit of which part of the optical laser light and visible synchrotron radiation from the same electron bunch are simultaneously focused. In the near future, the optical laser will be locked to the new synchronisation system, and the new bunch arrival time monitors should further improve the measurement of the relative timing to a precision of close to $10 \mathrm{fs}$.

Also the third route, the generation of two strictly synchronised light pulses, has been pursued since the planning phase of the FLASH facility. In 2001 it was proposed to add a ten-period electromagnetic undulator behind the FEL in order to produce strictly synchronised, intense coherent FIR pulses [21]. Such a device (with nine undulator periods) has been installed in 2007, together with a beamline and an integrated variable delay line that transports the FIR radiation to the BL3 station where it can be combined with the FEL beam and the optical laser [18] (see also section 3 below). Another approach for achieving the highest possible time resolution in a pump-probe experiment is splitting the FEL pulse in two parts, delaying one of them and recombining the two pulses on the sample. Two such devices have been built and integrated in the photon beamlines, one in BL2 with a delay range of -3 ps to +20 ps [22], the other with \pm 6 ps in PG2 [23]. The schematic layout of the former device is shown in figure 4. Finally, also seeding is a method providing perfect synchronisation between the optical seed laser and the FEL (see section 4 below). 


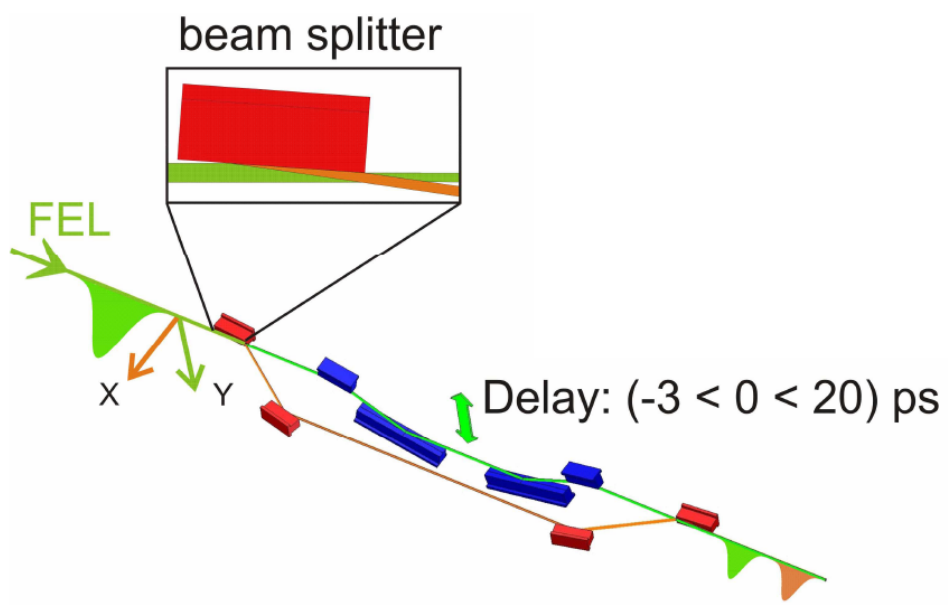

Figure 4. Schematic layout of the beam splitter installed in beam line BL2. Grazing angles of $3^{\circ}$ and $6^{\circ}$ for the fixed and variable delay arms, respectively, are employed to ensure a high reflectivity of the soft x-ray radiation [22].

\section{Photon diagnostics}

The characteristic features of the FEL photon pulses such as pulse energy, spectral and temporal structure, must be monitored in order to tune and operate the FEL as well as to perform and interpret the experiments. Since each pulse is different due to the stochastic nature of the SASE (self-amplified spontaneous emission) process by which the FEL pulses are produced, special diagnostics has been developed that allows shot-to-shot characterisation of the beam. A novel gas monitor detector based on the photoionisation of inert gases was developed in order to measure the energy of each pulse with an absolute precision of $\sim 10 \%[24,25]$. This detector covers the full spectral range of FLASH as well as the full dynamic range from spontaneous undulator radiation to FEL pulses in deep saturation. Due to the low gas pressure required, the FEL beam is not noticeably affected. Detectors of this type have been integrated in the photon transport system at the end of the accelerator tunnel and the entrance of the experimental hall before the FEL beam is deflected by the first mirror.

So far, the spectral distribution of single FEL pulses at FLASH has been measured routinely using a spectrometer at the end of the accelerator tunnel [26] or the plane grating monochromator [15]. In both instruments the footprint of the dispersed beam on a fluorescent screen in the exit slit plane can be recorded by a CCD camera. For many experiments, however, one would like to measure the spectral distribution of the FEL pulse that is actually used for the experiment. Therefore, a special spectrometer has been designed and integrated in the beam transport system such that it can be used for all BL beamlines [11, 27]. The concept (Figure 5) relies on the idea to replace the plane mirror that deflects the FEL beam to the left into beam lines BL1-3 by a plane grating with varied line spacing (VLS). The VLS grating acts as a mirror reflecting $~ 90 \%$ of the incoming light to the experiment. A small fraction of light is dispersed and focused (by means of the appropriately varied line spacing along the grating) on a fluorescent screen $\sim 2 \mathrm{~m}$ away from the grating. The screen can be moved along the focal curve and is imaged by a CCD camera. In the future, it is planned to replace this detection system by a fast array detector that can cope with the MHz pulse repetition rate of FLASH. The spectrometer will be taken into operation in the next months. 


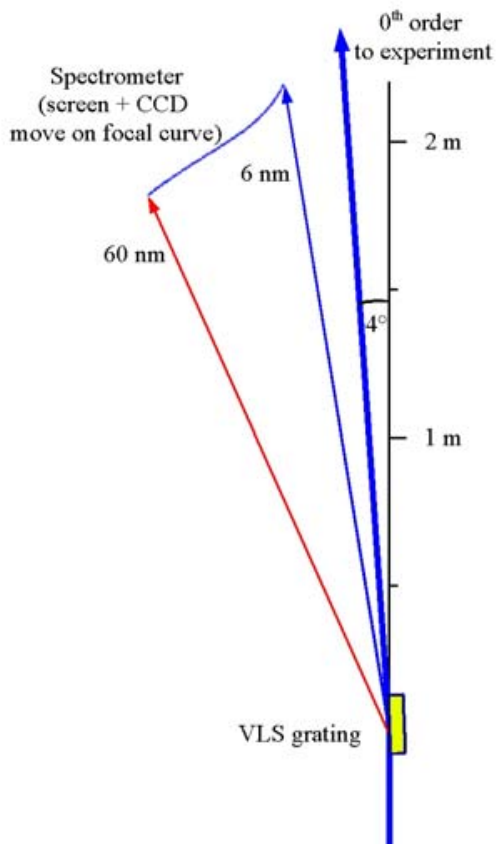

Figure 5. Concept of the VLS (varied line spacing) grating spectrometer for measuring the spectral distribution of single FEL pulses in a nondestructive way. $90 \%$ of the radiation is reflected to the experiment; a small amount is dispersed and focused on a fluorescent screen that is imaged by a CCD camera.

The fluctuating spectral distribution indicates that also the instantaneous intensity distribution along a FEL pulse fluctuates from pulse to pulse. This temporal structure must be known in order to understand in detail the nonlinear response of matter to the FEL excitation. So far it has not been possible to measure this structure directly. In a first attempt, the beam splitters described in section 3 were used for autocorrelation measurements [22, 23, 28]. When the two parts of the pulses overlap, interference fringes are observed (Figure 6). From the delay range for which the interference fringes are visible, a coherence time of $6 \mathrm{fs}$ was determined at $23.9 \mathrm{~nm}$ wavelength. At this wavelength, also the pulse duration of $29 \pm 5 \mathrm{fs}$ was directly measured by time-resolved observation of doubly charged helium ions [28]. It should be noted, however, that the autocorrelation measurements require scanning the delay between the two partial beams and thus average over many pulses.

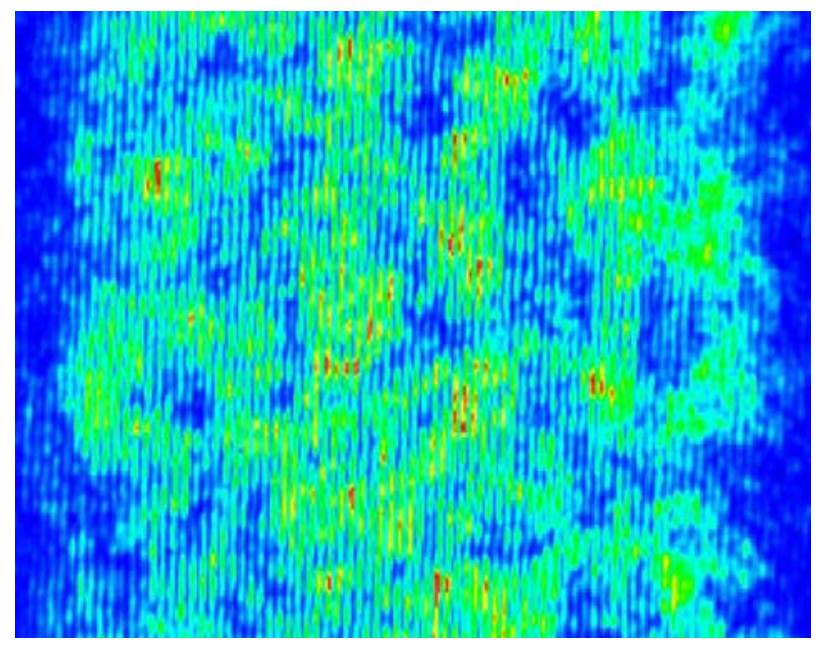

Figure 6. Interference fringes for the fully overlapped partial beams at $23.9 \mathrm{~nm}$ wavelength [22]. 
Recently, first promising experiments were made at FLASH to measure the temporal structure of single FEL pulses directly by using a few-femtosecond X-ray streak camera [29]. It was realized on BL3 by combining the FEL beam and the intense beam of the new FIR undulator described above. When matter it is ionized by the soft X-ray radiation of FLASH in the presence of the intense synchronized terahertz field of the FIR radiation, the kinetic energy of the photoelectrons can be changed significantly depending on amplitude and phase of the field at the time when the photoelectron is created. Since FEL and FIR pulse are generated by the same electron bunch, they are strictly synchronous and, therefore, the delay between these pulses can be adjusted by an optical delay line with an accuracy and stability of a few femtoseconds. In the first experiments krypton $4 p$ electrons were ionized with $13.5 \mathrm{~nm}$ radiation from FLASH and the FIR undulator was tuned to $92 \mu \mathrm{m}$ wavelength. Figure 7 shows a series of $\mathrm{Kr} 4 \mathrm{p}$ photoelectron spectra (vertical axis) at different delays (horizontal axis), sampling the terahertz field of the FIR pulse. The analysis of the data revealed a positive spectral chirp along the FEL pulses and distinct substructure for $10 \%$ of the pulses. The average pulse duration was determined at $35 \pm 7 \mathrm{fs}$ [29].

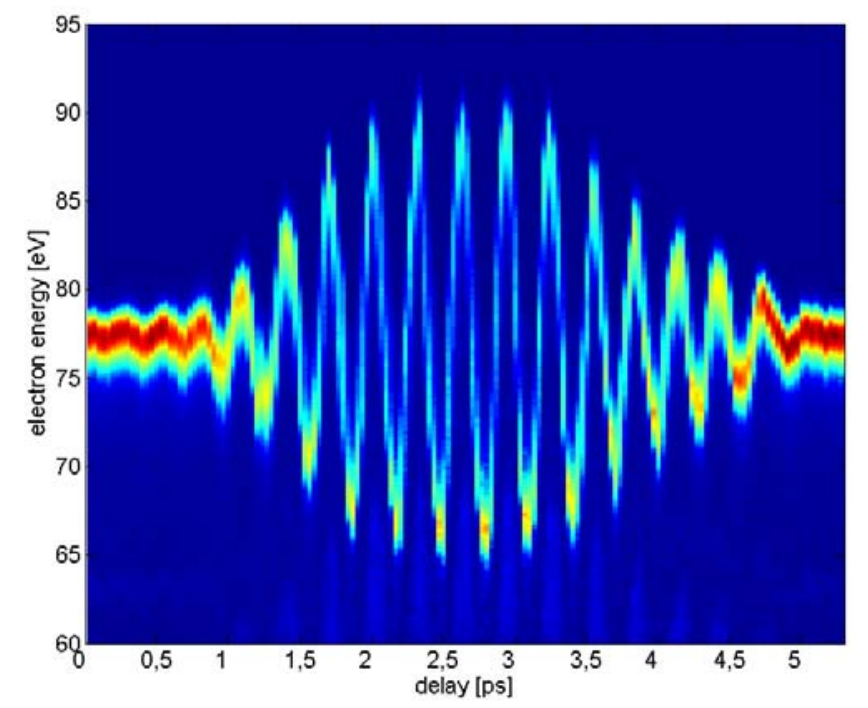

Figure 7. Series of kinetic energy spectra of krypton $4 p$ photoelectrons produced by a $13.5-n m$ FLASH pulse in the presence of an intense pulsed terahertz field. The energy shift of the electrons versus the X-ray/terahertz delay directly represents the vector potential of the THz field.

\section{Facility upgrades}

The FLASH facility has been continuously improved and two major upgrades have been made since the beginning of user operation in 2005. The main objectives of the first upgrade in 2007 were the installation of a sixth accelerator module with high-gradient cavities to reach the design energy of 1 $\mathrm{GeV}$, corresponding to $6.5 \mathrm{~nm}$ wavelength, and of the FIR undulator together with the complete beam line transporting the terahertz radiation to the BL3 experimental station. The second upgrade in 2009/10 included, among others, the installation of (i) a seventh accelerator module to further increase the energy and to achieve wavelengths below $5 \mathrm{~nm}$, i.e. close to the carbon K-edge, (ii) an accelerator section with four third-harmonic cavities to improve the bunch compression and allow forming highcurrent electron bunches of variable length up to several hundred femtoseconds, (iii) large parts of the optical synchronisation system, and (iv) all the components for seeding an additional FEL with high harmonic radiation of an optical laser generated in a gas target (HHG). 


\subsection{HHG seeding project}

The main motivation for seeding a FEL is to avoid the fluctuations in the temporal and spectral intensity distribution from pulse to pulse which are characteristic of SASE. Generating the seed radiation by an optical laser has the additional advantage of strict synchronisation between seed laser pulse and FEL output pulse such that a time resolution in pump-probe experiments can be achieved that is no longer determined by timing jitter but only by the duration of the pulses. Although all soft Xray FEL projects worldwide plan to use seeding, there is still a debate which technique is best suited. Three methods have been proposed to generate well-defined, transform limited FEL pulses with wavelengths down to $\sim 1 \mathrm{~nm}$ and durations of a few tens of femtoseconds: (i) high-gain harmonic generation (HGHG) using several cascades together with the so-called fresh-bunch technique, where the first stage is seeded by an optical laser in the visible or ultraviolet [30]; (ii) direct seeding with HHG laser pulses; (iii) echo-enabled harmonic generation (EEHG) where two optical seed lasers and two modulators are used in combination with two dispersion sections to generate a high harmonic density modulation in the electron bunch [31]. Apart from technical challenges, there is the fundamental problem of noise degradation if high harmonics and several cascades need to be employed to reach short wavelengths [32, 33]. In this regard, HHG seeding might be better than HGHG, and, from the technical point of view, the recently suggested EEHG looks even more attractive.

So far, there is no experience with cascading harmonic generation stages, and HHG seeding has only been demonstrated at long wavelengths, for the first time at $160 \mathrm{~nm}$ wavelength at SCSS (SPring-8 Compact SASE Source test accelerator) in Japan [34]. The HHG seeding project at FLASH (also referred to as sFLASH) is the first attempt to seed a FEL at high harmonics near $30 \mathrm{~nm}$ wavelength and to integrate it in a user facility. The technical design of sFLASH has been described in detail in reference [35]. A top view of the installations at FLASH is shown in figure 8. The HHG seed radiation is produced by a commercial $10 \mathrm{~Hz}$ laser system in a separate hutch outside the accelerator tunnel. It is transported into the tunnel and merged with the electron beam using multilayer-coated optics. Four variable-gap undulators, $10 \mathrm{~m}$ long in total, amplify the nanojoule seed pulses to saturation. The output radiation is transported to another hutch where it can be characterized and combined with part of the HHG pump laser source for pump-probe experiments. sFLASH is considered an important step towards extending the FLASH facility by a second, seeded FEL. In order to take full advantage of the high repetition rate of the superconducting accelerator in the future, the HHG source is further developed, in parallel to sFLASH, based on the new high repetition rate, few-cycle laser mentioned above [19]. The goal is to produce seed pulse trains with $10 \mu$ sulse distance (i.e. $100 \mathrm{kHz}$ bursts) at $10 \mathrm{~Hz}$ repetition rate in the first step, and then continue laser developments in collaboration with European XFEL and Helmholtz Institute Jena to reach MHz rates in the second step.

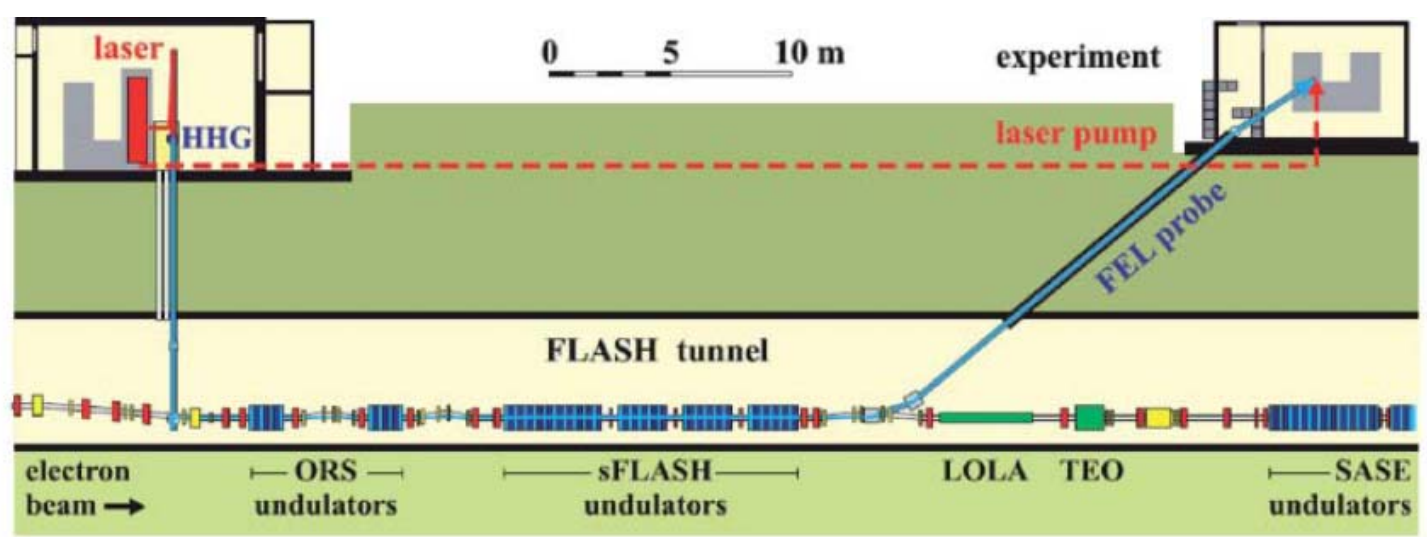

Figure 8. Top view of the HHG seeding installations at FLASH. 


\subsection{FLASH II extension}

The next major upgrade will be the construction of a second FEL undulator in a separate tunnel together with a second experimental hall. FLASH II, as the extension has been called, has three objectives: First, it will help accommodating the increasing demand for beam time by at least doubling the capacity for scientific experiments. Second, it will offer new opportunities for science applications due to increased beam quality by seeding, variable polarisation and an extended wavelength range down to $<4 \mathrm{~nm}$ in the fundamental and $\sim 1.3 \mathrm{~nm}$ in the $3^{\text {rd }}$ harmonic. Third, it will enable further technical developments such as new seeding schemes in a more efficient way and with less interference with user operation.

The schematic layout of this so-called FLASH II extension is shown in figure 9. The electron beam will be delivered by the existing FLASH accelerator. Similar to the electron beam distribution area planned for the European XFEL facility, an electron beam switch will be installed immediately behind the last accelerator module. Thus, the electron beam can be injected into the existing undulator beam line of FLASH, producing radiation for users in the present experimental hall with its five photon beam lines, and it can be intermittently deflected into the second undulator beam line FLASH II, which would then produce radiation for users in the new experimental hall which can accommodate another six experimental stations. The wavelengths can be adjusted independently because the FLASH II undulators will have a variable gap. In such a way, practically all users can get the maximum amount of pulses with the corresponding pulse pattern and pulse length that suits their specific experiment, thus making optimum use of the superconducting accelerator and serving a maximum number of users.

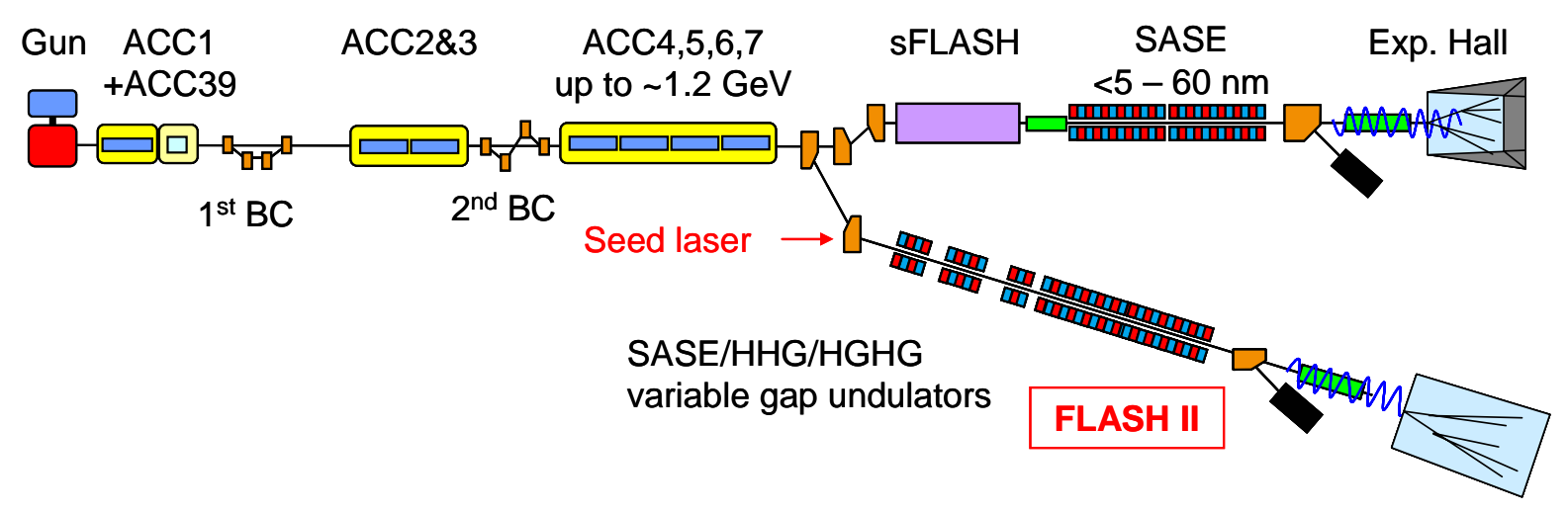

Figure 9. Schematic layout of the FLASH facility with the FLASH II extension on the lower right.

\section{Conclusions}

The FLASH facility as well as the scientific experiments have made enormous progress since the start of user operation in 2005. The technical operation of the facility, including the accelerator, the photon beam lines, diagnostics, laser systems and experiments, has become routine, along with all the associated administrative and organisational issues. Continuous technical improvements and two major upgrades have significantly enhanced the performance of the light source in terms of stability, wavelength range and additional instrumentation available for experiments such as synchronised optical lasers, an intense terahertz source, beam splitters, a high-resolution Raman spectrometer and advanced diagnostics. Intense research and development on electron beam dynamics, synchronisation and laser sources have paved the way for the integration of a HHG seeding experiment in the existing facility and an extension by a second seeded FEL (FLASH II) that is now in preparation. 
The technical developments of the FLASH facility have benefited largely from international collaborations. Some of them go back to TTF days, many have been established later, often as ad-hoc collaborations based on mutual interest and partially triggered and supported by national or European grants. In recent years these technical developments have been more systematically coordinated on a European level with funding from the Framework Programmes of the European Commission. The first initiative of this kind was the EUROFEL project where 16 partner institutions joined forces to develop critical technology required for constructing a set of new X-ray FEL sources in Europe [36]. On the political level and in parallel to these technical efforts, a European roadmap of new research infrastructures with a truly European dimension has been established by the European Strategy Forum on Research Infrastructures (ESFRI) [37]. The European XFEL as a large single-site facility is on this roadmap as well as EuroFEL as a so-called distributed European research infrastructure, i.e. a consortium of national FEL facilities including FLASH, FERMI@Elettra in Trieste, Italy, which is under construction, and other FEL projects in preparation. These infrastructures are joining forces to construct and operate an ensemble of most advanced free electron laser light sources for a broad, multidisciplinary user community.

\section{References}

[1] Åberg T et al., A VUV Free Electron Laser at the TESLA Test Facility at DESY, Conceptual Design Report TESLA FEL Report 1995-03 DESY 1995

[2] Schneider J R 2010 J. Phys. B: At. Mol. Opt. Phys.

[3] Ayvazyan V et al. 2006 European Physical Journal D 37297

[4] Ackermann W et al. 2007 Nat Photon 1336

[5] Bostedt C et al. 2009 Nucl. Instrum. Methods A 601108

[6] Henke B L, Gullikson E M and Davis J C 1993 At. Data Nucl. Data Tables 54181

[7] Lee R W et al. 2003 J. Opt. Soc. Am. B-Opt. Phys. 20770

[8] Steeg B, Juha L, Feldhaus J, Jacobi S, Sobierajski R, Michaelsen C, Andrejczuk A and Krzywinski J 2004 Applied Physics Letters 84657

[9] Hau-Riege S P et al. 2007 Applied Physics Letters 90173128

[10] Hau-Riege S P et al. 2009 Applied Physics Letters 95111104

[11] Tiedtke K et al. 2009 New Journal of Physics 11023029

[12] Sorokin a A, Bobashev S V, Feigl T, Tiedtke K, Wabnitz H and Richter M 2007 Physical Review Letters 99213002

[13] Nelson a J et al. 2009 Optics Express 1718271

[14] Martins M, Wellhöfer M, Hoeft J T, Wurth W, Feldhaus J and Follath R 2006 Review of Scientific Instruments 77115108

[15] Wellhöfer M, Martins M, Wurth W, Sorokin a A and Richter M 2007 Journal of Optics A Pure and Applied Optics 9749

[16] Will I, Redlin H, Düsterer S, Feldhaus J and Plönjes E Proceedings FEL2005 Stanford, USA.

[17] Radcliffe P et al. 2007 Nucl. Instrum. Methods A 583516

[18] Gensch M et al. 2008 Infrared Physics \& Technology 51423

[19] Tavella F et al. 2010 Optics Express 184689

[20] Schulz S et al. Proceedings FEL2009 Liverpool, UK.

[21] Faatz B, Fateev a A, Feldhaus J, Krzywinski J, Pflüger J, Rossbach J, Saldin E L, Schneidmiller E A and Yurkov M V 2001 Nuclear Instruments and Methods in Physics Research Section A: Accelerators, Spectrometers, Detectors and Associated Equipment 475363

[22] Mitzner R et al. 2008 Optics Express 1619909

[23] Schlotter W F, Sorgenfrei F, Beeck T, Beye M, Gieschen S, Meyer H, Nagasono M, Föhlisch A and Wurth W 2010 Optics Letters 35372

[24] Richter M et al. 2003 Applied Physics Letters 832970

[25] Tiedtke K et al. 2008 Journal of Applied Physics 103094511 
[26] Nicolosi P, Poletto L, Pelizzo M G, Epulandi L, Zambolin P, Feldhaus J, Jastrow U, Hahn U, Plönjes E and Tiedtke K 2005 Journal of Electron Spectroscopy and Related Phenomena 144 1055

[27] Reininger R, Feldhaus J, Plönjes E, Treusch R, Roper M D, Quinn F M and Bowler M A 2004 AIP Conference Proceedings 705572

[28] Mitzner R et al. 2009 Physical Review A 80025402

[29] Frühling U et al. 2009 Nat Photon 3523

[30] Ben-Zvi I, Yang K M and Yu L H 1992 Nucl. Instrum. Methods A 318726

[31] Xiang D and Stupakov G 2009 Physical Review Special Topics-Accelerators and Beams 12 030702

[32] Saldin E L, Schneidmiller E A and Yurkov M V 2002 Optics Communications 202169

[33] Sheehy B, Clarke J A, Thompson N R, McNeil B W J and Gordon A 2008 Nucl. Instrum. Methods A 59321

[34] Lambert G et al. 2008 Nature Physics 4296

[35] Miltchev V et al. Proceedings FEL2009 Liverpool, UK.

[36] Feldhaus J, Ferianis M, Ferrario M, Knobloch J, Krell U, Limberg T, Owen H, Petersen B and Werin S 2008 Synchrotron Radiation News 2128

[37] ESFRI, European Roadmap for Research Infrastructures - Report 2006 and Roadmap 2008 